\title{
Computed tomography imaging of resuscitative endovascular balloon occlusion of the aorta (REBOA): pearls and pitfalls
}

\author{
Ryo Aoki ${ }^{1,2} \cdot$ Yusuke Kobayashi $^{2} \cdot$ Shintaro Nawata ${ }^{2} \cdot$ Hiroyuki Kamide $^{2} \cdot$ Toh Yamamoto $^{2} \cdot$ Shintaro Furugori $^{3}$. \\ Zenjiro Sekikawa $^{2} \cdot$ Daisuke Utsunomiya $^{1}$
}

Received: 22 March 2021 / Accepted: 21 June 2021 / Published online: 3 July 2021

(c) The Author(s) 2021

\begin{abstract}
Resuscitative endovascular balloon occlusion of the aorta (REBOA) is performed in patients with hemorrhagic shock who develop massive subdiaphragmatic bleeding. This procedure enables rapid and less invasive aortic blockade compared to resuscitative thoracotomy and aortic cross-clamp procedures. However, the REBOA procedure is often blindly performed in the emergency department without fluoroscopy, and the appropriateness of the procedure may be evaluated on computed tomography (CT) after REBOA. Therefore, radiologists should be familiar with the imaging features of REBOA. We present a pictorial review of the radiological findings of REBOA along with a description of the procedure, its complications, and pitfalls.
\end{abstract}

Keywords Resuscitative endovascular balloon occlusion of the aorta $\cdot$ Computed tomography $\cdot$ Complications $\cdot$ Pitfalls

\section{Introduction}

High-energy trauma is defined as open or closed injuries caused by forces (e.g., motor vehicle accidents). Organs and tissues can sustain extensive damage due to a high amount of kinetic energy, causing severe bleeding. In such timesensitive and life-threatening situations, urgent endovascular techniques are essential [1].

Resuscitative endovascular balloon occlusion of the aorta (REBOA) is a resuscitation procedure in which a balloon catheter is inserted into the aorta to create a balloon blockade that increases proximal arterial pressure to maintain central organ perfusion while controlling distal subdiaphragmatic hemorrhage [2]. Aortic blockade with REBOA is less invasive than resuscitative thoracotomy

Ryo Aoki

aokir223@yahoo.co.jp

1 Diagnostic Radiology, Yokohama City University Graduate School of Medicine, Yokohama-shi, 4-57 Urafunecho, Minami-ku, Yokohama-shi, Kanagawa 232-0024, Japan

2 Diagnostic Radiology, Yokohama City University Medical Center, Yokohama-shi, Kanagawa, Japan

3 Advanced Critical Care and Emergency Center, Yokohama City University Graduate School of Medicine, Yokohama-shi, Kanagawa, Japan and aortic cross-clamp procedures [3]. Ideally, the REBOA procedure is performed under guidance by fluoroscopy and/ or ultrasound for appropriate placement without complications. However, the REBOA procedure is highly emergent and may be performed blindly because these modalities and human resources are often unavailable in emergency situations. Furthermore, the assessment by ultrasonography is operator-dependent. On the other hand, computed tomography $(\mathrm{CT})$ is advantageous because $\mathrm{CT}$ provides objective assessment, information on REBOA placement, and status of various organs. Therefore, radiologists should be familiar with the imaging features of REBOA. We present a pictorial review of the radiological findings of REBOA along with a description of its procedure, classification, complications, and pitfalls.

\section{REBOA procedure}

The procedure was performed using a 7-French REBOA device (Rescue Balloon ${ }^{\mathrm{TM}}$ or Rescue Balloon ER ${ }^{\mathrm{TM}}$, Tokai Medical Products Corp., Kasugai, Aichi, Japan) according to the following steps: (1) establish arterial access (usually through the common femoral artery considering ultrasound guidance or cut-down approach) and place the sheath, (2) insert the REBOA catheter (balloon catheter) over the 
guidewire, (3) remove the guidewire and insert the stylet into the REBOA catheter to avoid kinking or migration of the balloon catheter because of the arterial pressure during balloon inflation, and 4) inflate the balloon. Ideally, balloon proximal and distal arterial pressure monitoring lines (e.g., left radial and femoral artery) allow us to determine adequate balloon positioning and inflation [4].

\section{Types of REBOA based on balloon inflation}

A trade-off between the occlusion duration of REBOA and tissue ischemia exists. There are three types of balloon inflation techniques. The REBOA type is chosen according to the disease severity. To reduce the risk of tissue ischemia, partial or intermittent REBOA is performed $[5,6]$.

\section{- Complete REBOA}

Balloon inflation can result in the cessation of distal pulse pressure by total aortic occlusion. However, complete REBOA increases the risk of distal ischemia and cardiac afterload.

\section{- Partial REBOA}

The balloon is partially inflated, and the aorta is not completely occluded. However, the control of downstream bleeding may be incomplete.

\section{- Intermittent REBOA}

Repeated inflation and deflation of the balloon are performed to restore downstream perfusion. Intermittent REBOA is expected to improve survival while minimizing ischemia-reperfusion injury [6].

\section{Balloon positions and indications}

The balloon position is divided into three zones (Figs. 1, 2) [7].

\section{Zone I}

The balloon is positioned in the aorta between the origin of the left subclavian and celiac arteries. Zone I is indicated in cardiac arrest or hemorrhagic shock with intra-abdominal hemorrhage (e.g., severe trauma and rupture of an abdominal aortic aneurysm).

\section{Zone II}

Zone II is defined as the area between Zones I and III. Placement in Zone II should be contraindicated due to risk of gastrointestinal ischemia.

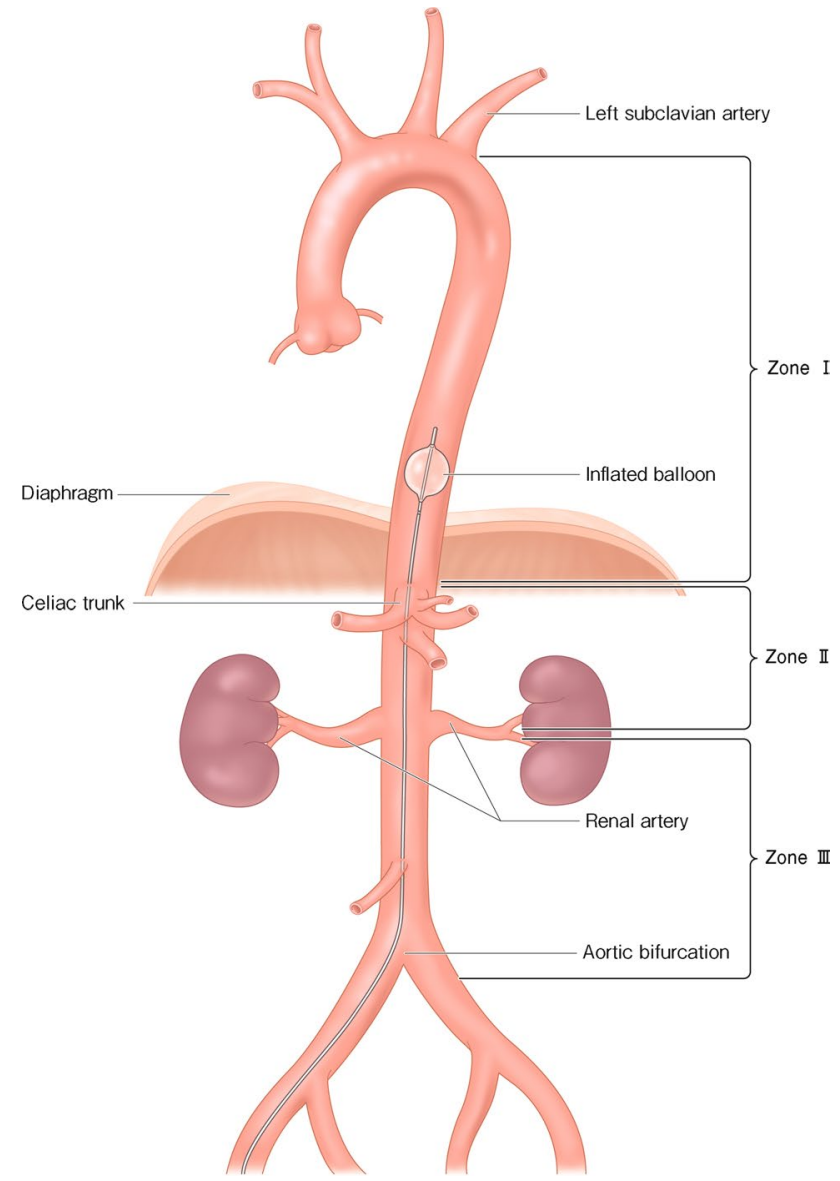

Fig. 1 The balloon inflation site is divided into three zones. Zone I is the area between the origin of the left subclavian artery and the celiac artery. Zone II is defined as the area between the lower end of Zone I and the top of Zone III. Zone III is the area between the origin of the lowest renal artery and the aortic bifurcation

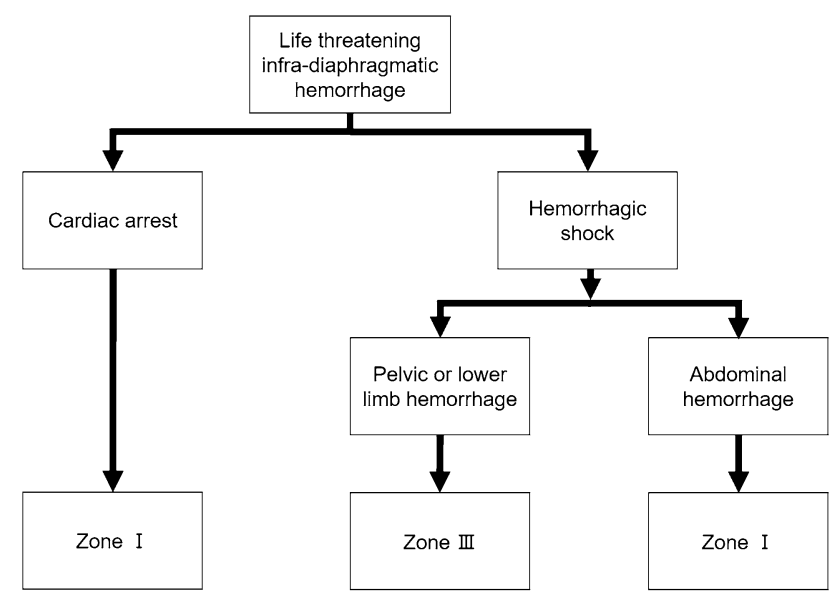

※ Zone II has no current indication.

Fig. 2 Algorithm showing the appropriate Zone for resuscitative endovascular balloon occlusion of the aorta 


\section{Zone III}

The balloon is positioned in the aorta between the origins of lowest renal artery to the aortic bifurcation. Zone III is indicated in patients with life-threatening pelvic or lower limb hemorrhage (e.g., pelvic fracture and postpartum hemorrhage).

\section{Evaluation of CT after REBOA}

Radiologists must assess the appropriateness of REBOA procedure (Figs. 3,4) and detect active bleeding on CT. In some patients, distal perfusion and active extravasation can still be detected in the setting of complete REBOA. It is speculated that the balloon and aortic wall were not watertight [8] with multiple collateral pathways from proximal to distal aorta [9].
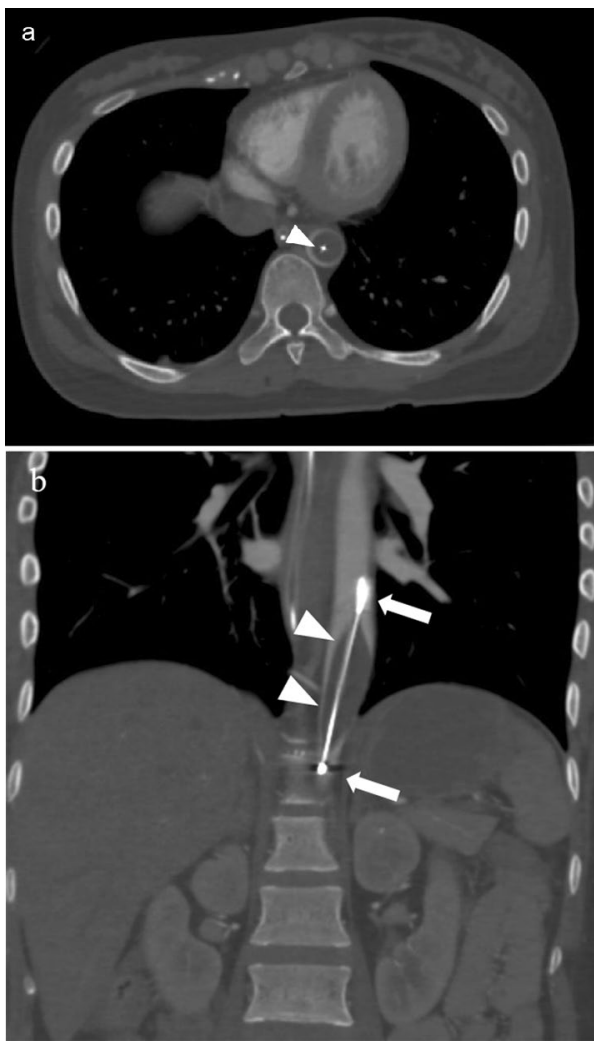

Fig. 3 Contrast-enhancement CT (a: axial, b: coronal view) shows an appropriate partial resuscitative endovascular balloon occlusion of the aorta placed in Zone I. The white arrows represent radiopaque markers of the balloon catheter, and the white arrowheads represent the stylet in the balloon catheter

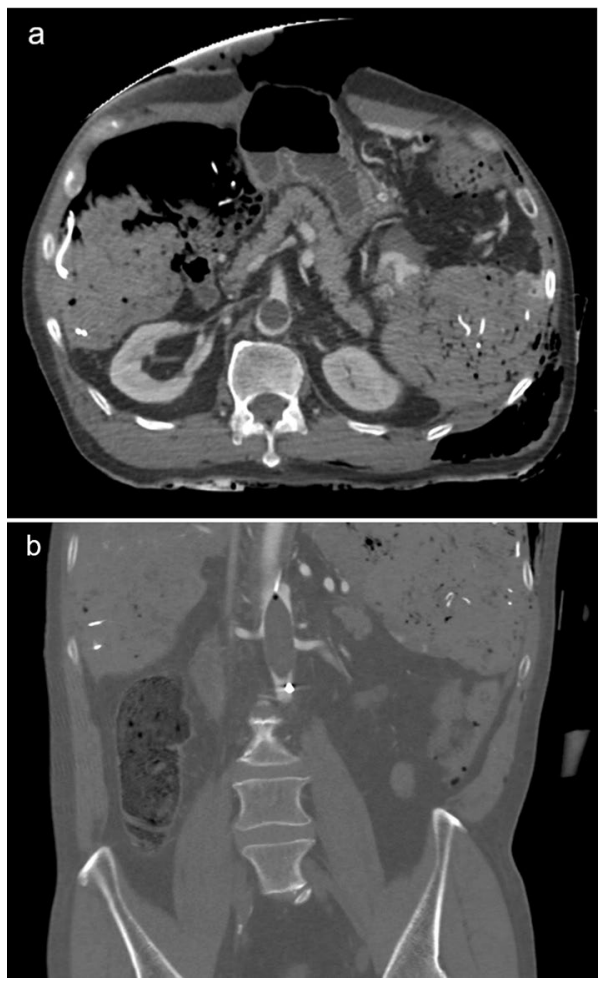

Fig. 4 Contrast-enhancement computed tomography shows an inappropriate resuscitative endovascular balloon occlusion of the aorta for a patient with multiple trauma (a: axial, b: coronal view). The balloon was positioned at Zone II, and the stylet was not inserted in the balloon catheter

\section{Complications}

There are two main types of complications: perfusionrelated and procedure-related complications.

\section{Perfusion-related complications}

Perfusion-related complications include distal ischemia-reperfusion injury and exacerbation of proximal bleeding.

\section{Organ ischemia and reperfusion injury}

REBOA can control subdiaphragmatic hemorrhage by decreasing distal arterial pressure; however, it may decrease organ perfusion (Fig. 5). Ischemia and reperfusion injury are common [10]; thus, inflation time should be minimized to prevent irreversible ischemic organ injury. Ideally, total aortic occlusion time $<30$ min avoids ischemic complications [11]. 


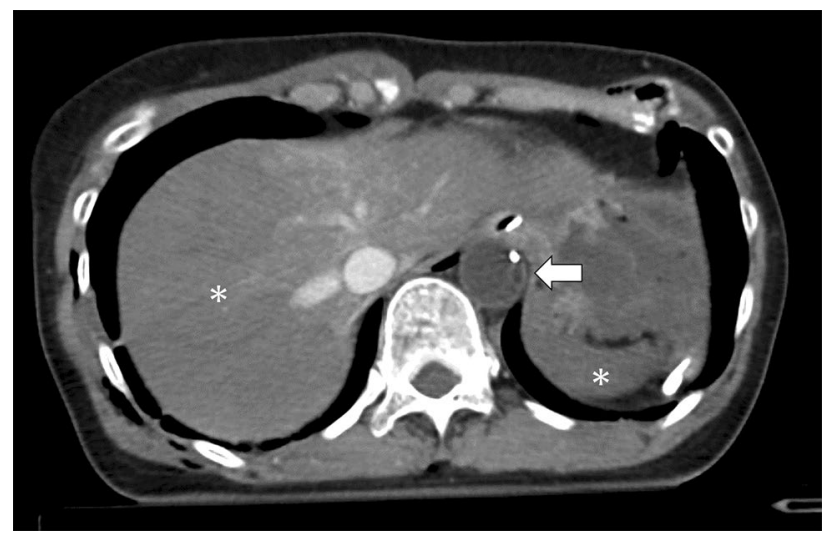

Fig. 5 Abdominal contrast-enhancement computed tomography (venous phase) with complete Zone I resuscitative endovascular balloon occlusion of the aorta (arrow) showing hypo-enhancement (hypoperfusion) in the liver and spleen (asterisks)
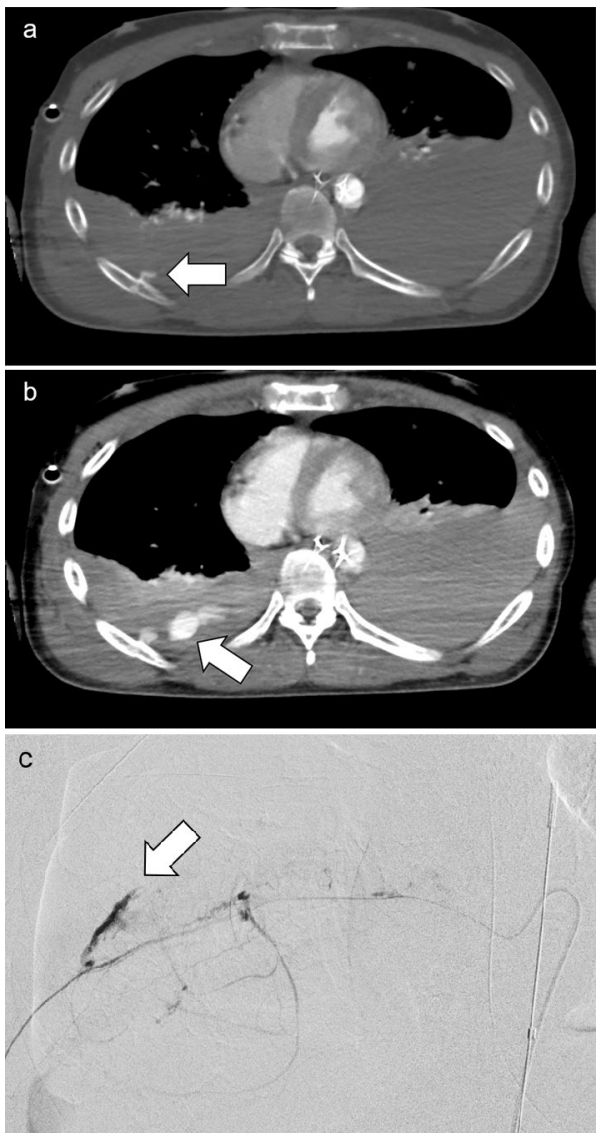

Fig. 6 Thoracic contrast-enhancement computed tomography (a: arterial phase, b: venous phase) with Zone I resuscitative endovascular balloon occlusion of the aorta under temporally deflated status after total inflation in the patient with multiple traumas showing extravasation (a and $\mathbf{b}$, arrow) from the right intercostal artery, which was confirmed by angiography (c, arrow). The bleeding was treated by transcatheter arterial embolization

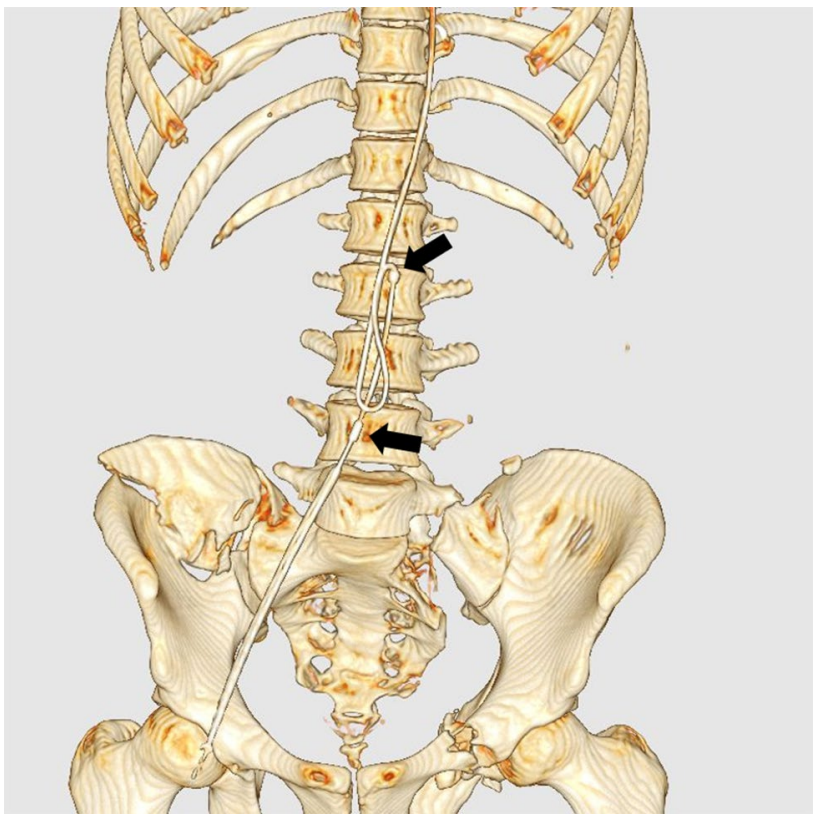

Fig. 7 Three-dimensional computed tomography showing loop formation of the guidewire. The balloon catheter could not be inserted due to the looping of the guidewire. The black arrows indicate the radiopaque markers of the balloon catheter

\section{Exacerbation of bleeding}

REBOA can worsen hemorrhage above the diaphragm [12] because of increased arterial pressure; therefore, the necessity of REBOA should be carefully evaluated (Fig. 6).

\section{REBOA-placement-related complications}

REBOA-placement-related complications include iatrogenic vessel injuries and inappropriate device insertion (e.g., kinking, loop formation, and migration).

\section{Loop formation}

The REBOA procedure can be performed blindly, which may cause loop formation of the guidewire (Fig. 7) or the balloon catheter shaft [13].

\section{Migration}

Unknown vessel injury or variant artery can potentially lead to device migration during the procedure (Fig. 8). Device migration may cause iatrogenic injury and render REBOA ineffective. 


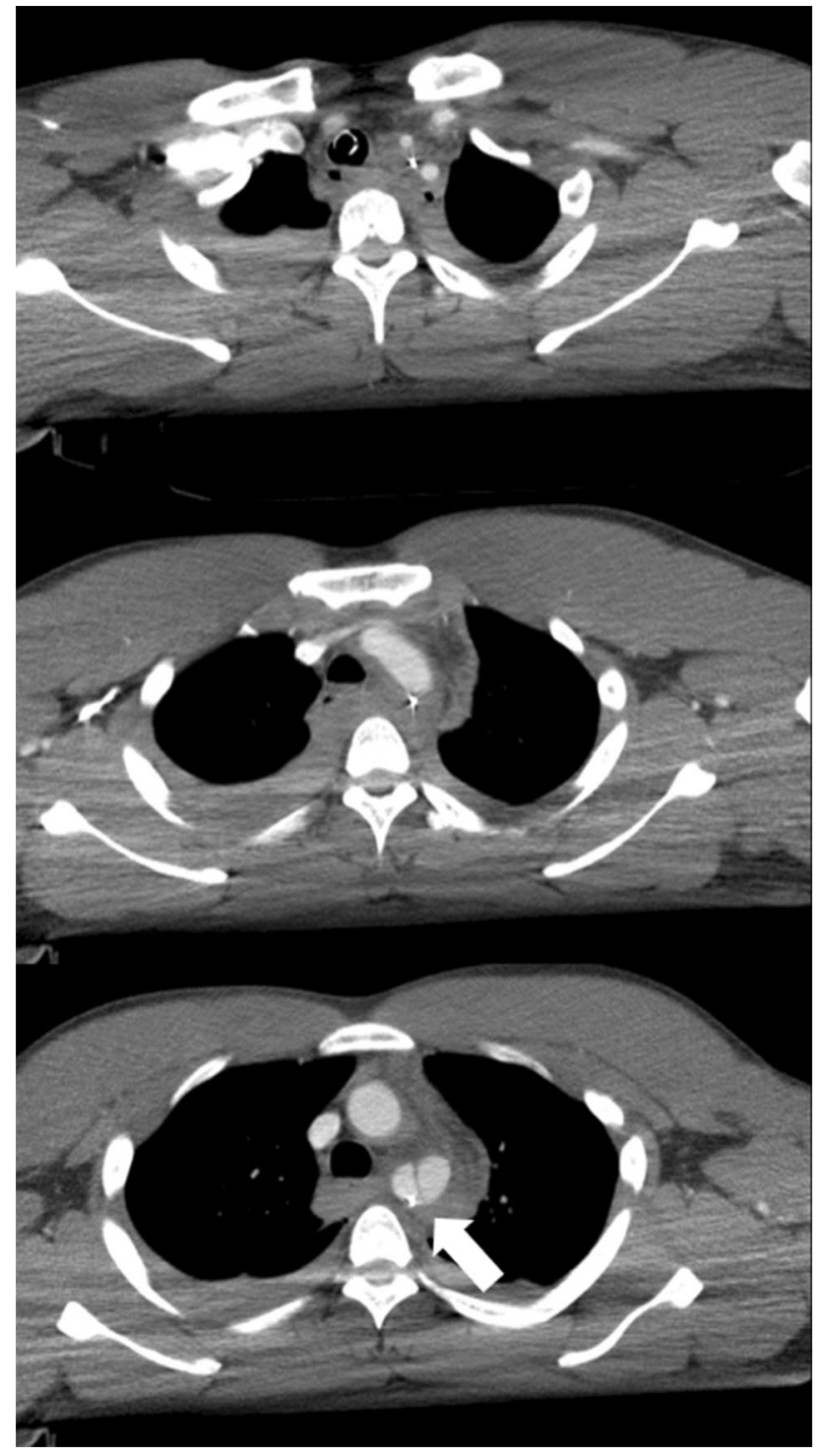

Fig. 8 Thoracic contrast-enhancement computed tomography in a patient with multiple traumas showing a complication of the guidewire migration into the arterial subintima (arrow). Resuscitative endovascular balloon occlusion of the aorta (REBOA) is contraindicated in a thoracic aortic injury, but the injury was not expected in the REBOA procedure in this case

\section{Pitfalls in CT interpretation}

\section{Hidden extravasation}

Although REBOA can control subdiaphragmatic hemorrhage, hidden extravasation of the contrast medium may occur (Fig. 9). The absence of extravasation on contrastenhanced CT for REBOA cannot rule out downstream bleeding.

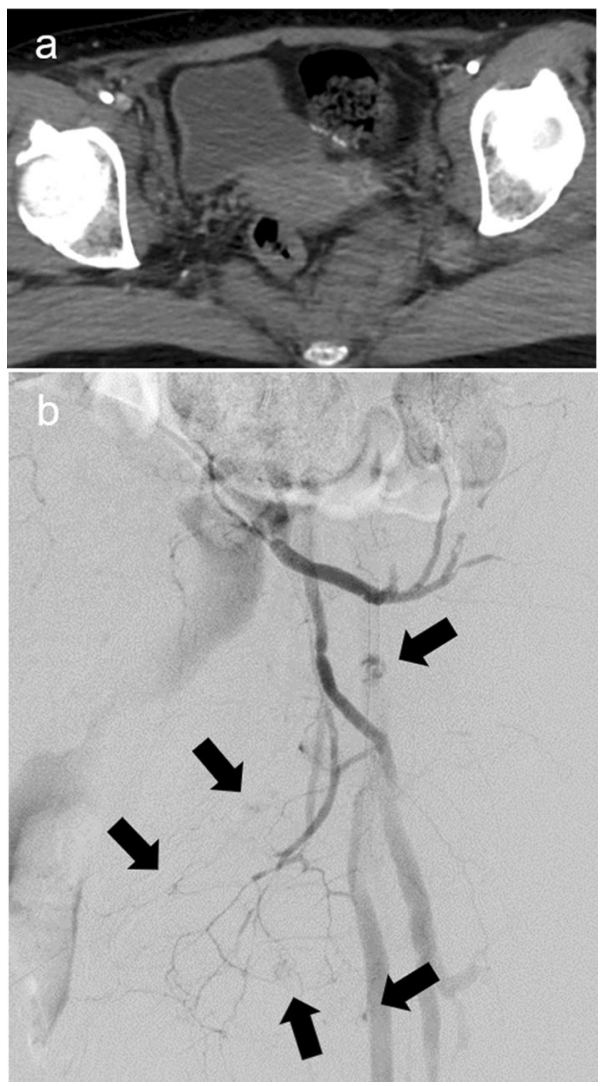

Fig. 9 Pelvic contrast-enhancement computed tomography (CT) with complete resuscitative endovascular balloon occlusion of the aorta (REBOA) in Zone I in a patient with pelvic fractures showing a massive hematoma in the retroperitoneal space without extravasation. However, left internal iliac angiography (REBOA was deflated temporarily) shows diffuse extravasation (black arrows), which was hidden by the decreased blood flow under complete REBOA during CT scanning

\section{Differentiation of organ injury and ischemia}

Renal contusions or renal vascular injury are characterized by decreased enhancement of the renal parenchyma [14]. Renal injury sometimes mimics the renal ischemia caused by REBOA (Figs. 10,11). The presence of injury around the kidney may be a clue in distinguishing between renal injury and ischemia.

\section{Presence of baseline aortic disease}

The medical history may be uncertain in some emergency patients. A history of aortic diseases (e.g., aortic aneurysm, aortic dissection, and postoperative state) may cause complications or render REBOA ineffective (Fig. 12). 

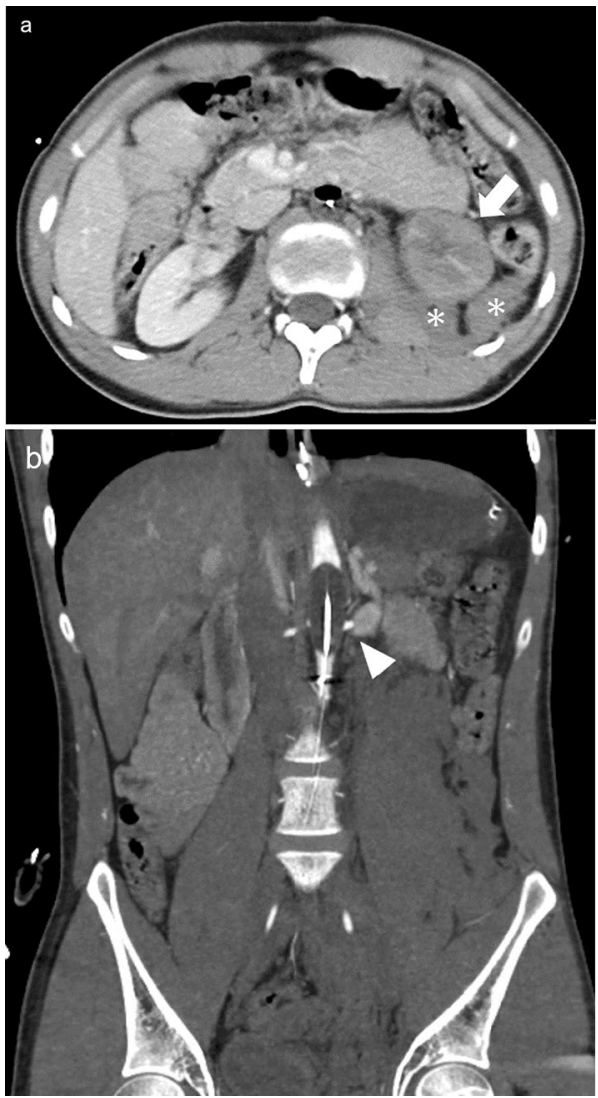

Fig. 10 Abdominal contrast-enhancement computed tomography (a: axial image, b: coronal view) with complete resuscitative endovascular balloon occlusion of the aorta (REBOA) in a patient with multiple traumas showing decreased enhancement on the left kidney (a, arrow) and hematoma around it (a, asterisks), which is suggestive of renal contusion. However, in this case, REBOA was misplaced in Zone II and the orifice of the left renal artery was occluded by the balloon (b, arrowhead). Therefore, it is difficult to conclude that this case was REBOA-related hypoperfusion or renal injuries

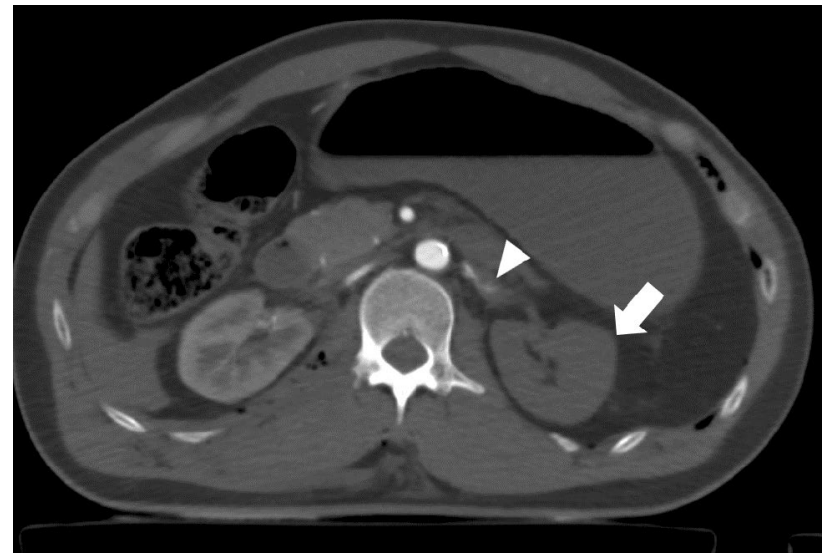

Fig. 11 Abdominal contrast-enhancement computed tomography with partial resuscitative endovascular balloon occlusion of the aorta in Zone $\mathrm{I}$ in a patient with multiple traumas showing no enhancement in the left kidney (arrow). The left renal artery was irregular and occluded (vascular injury) (arrowhead)
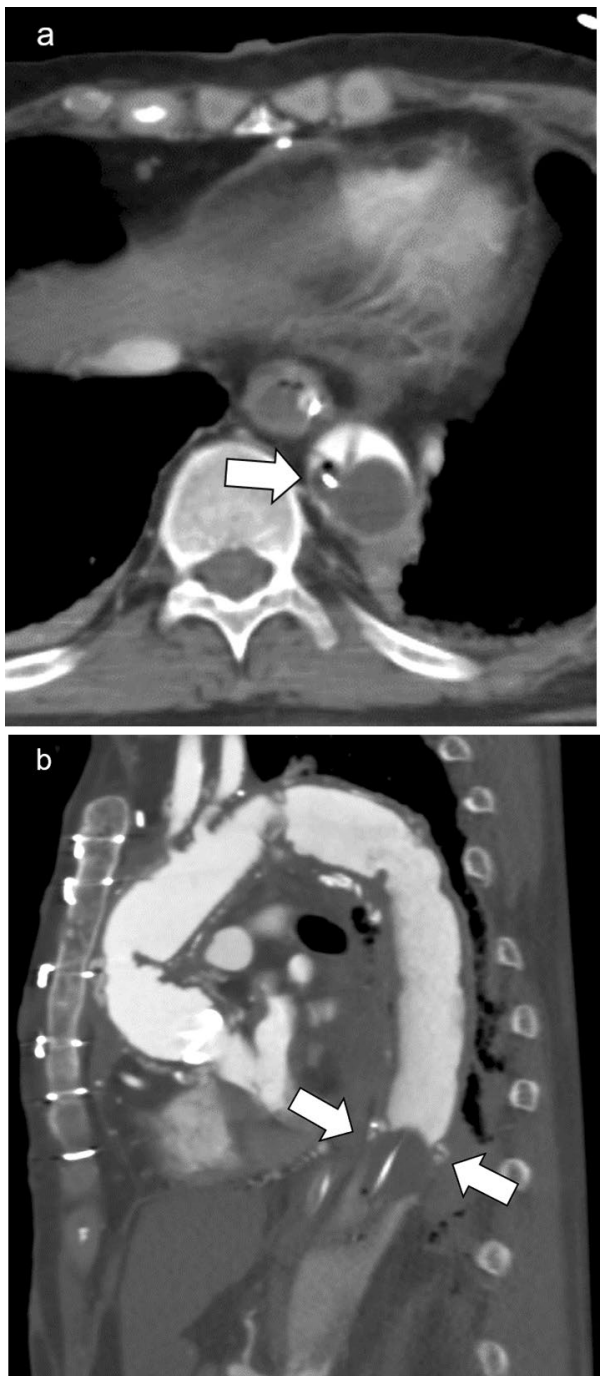

Fig. 12 Thoracic contrast-enhancement computed tomography (a: axial image, b: sagittal view) in a patient with prior aortic replacement for aortic dissection (Stanford type A) showing that the catheter shaft was positioned in the smaller lumen (true lumen) (a, arrow) and the balloon was inflated in the false lumen through the double-barreled anastomosis (b, arrows)

\section{Venous injuries}

The efficacy of REBOA in the presence of a major venous abdominal injury is unclear. REBOA appears to be effective for central venous injuries in a porcine model [15]; however, the utility of REBOA for venous injuries in humans with multiple traumatic injuries is unknown. Radiologists should highlight venous injuries to develop an appropriate treatment strategy (Fig. 13). 


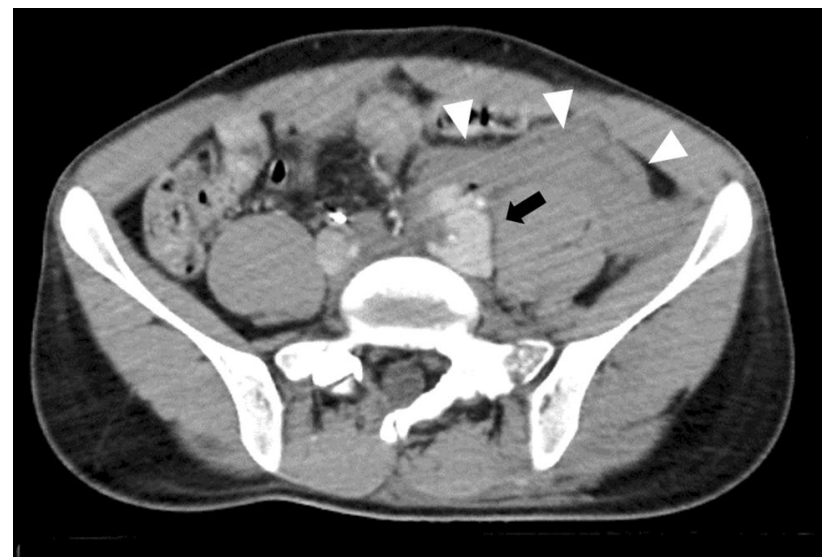

Fig. 13 Pelvic contrast-enhancement computed tomography with complete resuscitative endovascular balloon occlusion of the aorta in a patient with multiple trauma showing massive extravasation from the left external iliac vein (black arrow) with a massive hematoma (white arrowheads)

\section{Conclusion}

REBOA plays an important role in patients with severe hemorrhagic shock. Accurate and prompt interpretation of CT findings is essential for treatment and can be a life-saving maneuver.

Authors' contributions All authors read and approved the final manuscript.

Funding No funding or sponsorship was received for this study or publication of this article.

\section{Declarations}

Conflict of interest The authors declare that they have no conflict of interest.

Ethical statement This article does not contain any studies with human participants or animals performed by any of the authors. Personal information was removed from this article according to the privacy policy of Japan Radiological Society.

Open Access This article is licensed under a Creative Commons Attribution 4.0 International License, which permits use, sharing, adaptation, distribution and reproduction in any medium or format, as long as you give appropriate credit to the original author(s) and the source, provide a link to the Creative Commons licence, and indicate if changes were made. The images or other third party material in this article are included in the article's Creative Commons licence, unless indicated otherwise in a credit line to the material. If material is not included in the article's Creative Commons licence and your intended use is not permitted by statutory regulation or exceeds the permitted use, you will need to obtain permission directly from the copyright holder. To view a copy of this licence, visit http://creativecommons.org/licenses/by/4.0/.

\section{References}

1. Halvachizadeh S, Mica L, Kalbas Y, Lipiski M, Canic M, Teuben $\mathrm{M}$, et al. Zone-dependent acute circulatory changes in abdominal organs and extremities after resuscitative balloon occlusion of the aorta (REBOA): an experimental model. Eur J Med Res. 2021;26:10

2. Benham DA, Calvo RY, Carr MJ, Wessels LE, Schrader AJ, Lee $\mathrm{JJ}$, et al. Is cerebral perfusion maintained during full and partial reboa in hemorrhagic shock conditions? J Trauma Acute Care Surg. 2021. https://doi.org/10.1097/TA.0000000000003124.

3. Moore LJ, Brenner M, Kozar RA, Pasley J, Wade CE, Baraniuk MS, et al. Implementation of resuscitative endovascular balloon occlusion of the aorta as an alternative to resuscitative thoracotomy for noncompressible truncal hemorrhage. J Trauma Acute Care Surg. 2015;79:523-32.

4. Ogura T, Lefor AK, Nakamura M, Fujizuka K, Shiroto K, Nakano M. Ultrasound-guided resuscitative endovascular balloon occlusion of the aorta in the resuscitation area. J Emerg Med. 2017:52:715-22.

5. Matsumura Y, Matsumoto J, Kondo H, Idoguchi K, Ishida T, Kon $\mathrm{Y}$, et al. Fewer REBOA complications with smaller devices and partial occlusion: evidence from a multicentre registry in Japan. Emerg Med J. 2017;34:793-9.

6. Kuckelman J, Derickson M, Barron M, Phillips CJ, Moe D, Levine $\mathrm{T}$, et al. Efficacy of intermittent versus standard resuscitative endovascular balloon occlusion of the aorta in a lethal solid organ injury model. J Trauma Acute Care Surg. 2019;87:9-17.

7. King DR. Initial care of the severely injured patient. N Engl J Med. 2019;380:763-70.

8. Matsumura Y, Higashi A, Izawa Y, Hishikawa S, Kondo H, Reva $\mathrm{V}$, et al. Organ ischemia during partial resuscitative endovascular balloon occlusion of the aorta: Dynamic 4D Computed tomography in swine. Sci Rep. 2020;10:5680.

9. Wasicek PJ, Shanmuganathan K, Teeter WA, Gamble WB, Hu P, Stein DM, et al. Assessment of blood flow patterns distal to aortic occlusion using CT in patients with resuscitative endovascular balloon occlusion of the aorta. J Am Coll Surg. 2018;226:294-308.

10. Abid M, Neff LP, Russo RM, Hoareau G, Williams TK, Grayson JK, et al. Reperfusion repercussions: A review of the metabolic derangements following resuscitative endovascular balloon occlusion of the aorta. J Trauma Acute Care Surg. 2020;89:S39-44.

11. Bulger EM, Perina DG, Qasim Z, Beldowicz B, Brenner M, Guyette F, et al. Clinical use of resuscitative endovascular balloon occlusion of the aorta (REBOA) in civilian trauma systems in the USA, 2019: a joint statement from the American College of Surgeons Committee on Trauma, the American College of Emergency Physicians, the National Association of Emergency Medical Services Physicians and the National Association of Emergency Medical Technicians. Trauma Surg Acute Care Open. 2019;4:000376.

12. Uchino H, Tamura N, Echigoya R, Ikegami T, Fukuoka T. "REBOA" - is it really safe? a case with massive intracranial hemorrhage possibly due to endovascular balloon occlusion of the aorta (REBOA). Am J Case Rep. 2016;17:810-3.

13. Onishi Y, Kimura H, Kanagaki M, Oka S, Fukumoto G, Otani $\mathrm{T}$, et al. Loop formation by an aortic occlusion balloon catheter during resuscitative endovascular balloon occlusion of the aorta (REBOA). Radiol Case Rep. 2019;14:184-6.

14. Harris AC, Zwirewich CV, Lyburn ID, Torreggiani WC, Marchinkow LO. Ct findings in blunt renal trauma. Radiographics. 2001;21:201-14. 
15. Lallemand MS, Moe DM, McClellan JM, Smith JP, Daab L, Marko S, et al. Resuscitative endovascular balloon occlusion of the aorta for major abdominal venous injury in a porcine hemorrhagic shock model. J Trauma Acute Care Surg. 2017;83:230-6.
Publisher's Note Springer Nature remains neutral with regard to jurisdictional claims in published maps and institutional affiliations. 